more frequently presented with B12 deficiency $(p=0.037)$ and had milder degrees of VA than younger patients $(\mathrm{p}=0.005)$.

Conclusions Coeliac disease is common in elderly patients but gastrointestinal symptoms occur less frequently than in younger individuals. Elderly patients tend to present with a milder degree of VA. This questions the utility of active case finding in this age group, as a gluten free diet may not be the most appropriate management in this cohort.

\begin{tabular}{|c|c|c|c|c|c|}
\hline & \multirow{3}{*}{$\begin{array}{l}\text { Prevalence in } \\
\text { overall prospective } \\
\text { cohort }(n=644)\end{array}$} & \multicolumn{3}{|c|}{ Age (years) } & \multirow[t]{3}{*}{$p$-value } \\
\hline & & $18-34$ & $35-64$ & $>65$ & \\
\hline & & $(n=259)$ & $(n=287)$ & $(n=99)$ & \\
\hline Fatigue & $24.9 \%(160)$ & $31.9 \%(82)$ & $23.0 \%(66)$ & $12.1 \%(12)$ & $<0.001$ \\
\hline Diarrhoea & $30.4 \%$ (196) & $35.7 \%(92)$ & $30.0 \%(86)$ & $18.2 \%(18)$ & 0.005 \\
\hline Abdominal pain & $23.2 \%(149)$ & $29.2 \%(75)$ & $20.2 \%(58)$ & $16.2 \%(16)$ & 0.019 \\
\hline $\begin{array}{l}\text { IBS-type } \\
\text { symptoms }\end{array}$ & $18.0 \%(528)$ & $24.4 \%(63)$ & $15.0 \%(43)$ & $10.1 \%(10)$ & 0.008 \\
\hline B12 deficiency & $12.1 \%$ (78) & $10.5 \%(27)$ & $10.8 \%(31)$ & $20.2 \%(20)$ & 0.037 \\
\hline
\end{tabular}

\section{PWE-035 GLOBAL TRANSLATION OF COELIAC DISEASE HISTOLOGY AND OTHER GLUTEN RELATED MICROENTEROPATHY}

Kamran Rostami, Mohammad Derakhshan*, Arzu Ensari, Amitabh Srivastava, Vincenzo Villanacci, Michael Marsh, Antonio Carroccio, Umberto Volta, Alessio Fasano, Julio Cesar Bai, Mihai Danciu, David Sanders, Anna Sapone, Carolina Ciacci, Luca Elli, Stefano Guandalini, Marjorie Walker, Laura De Magistris, Hilary Jericho, Sauid Ishaq, Gabriel Becheanu, Carlo Catassi, Sherly Mathews, James Going, Mohammad RostamiNejad, Chris Mulder, Hamid Mohaghegh, Matt Johnson, Geoffrey Holmes, Gabrio Bassotti, Anna Bozzola, Chiara Ricci, Ada Maria Florena, Rachele Delsordo, Roxana Maxim, Prasenjit Das, Govind Makharia, Knut Lundin, Katri Kaukinen, Adam Levene, Nicola Fusco, Afshin Moradi, Giovanni Casella, David Hayman, Camillo Dibella, Catherine Hagen, Giuseppe Mazzarella, Melanie Johncilla, Mehul Lamba, Juha Taavela, Mohammad Reza Zali, Sarah Liprot, Christine Rodger. Department of Gastroenterology, Palmerston North, New Zealand

10.1136/gutjnl-2019-BSGAbstracts.322

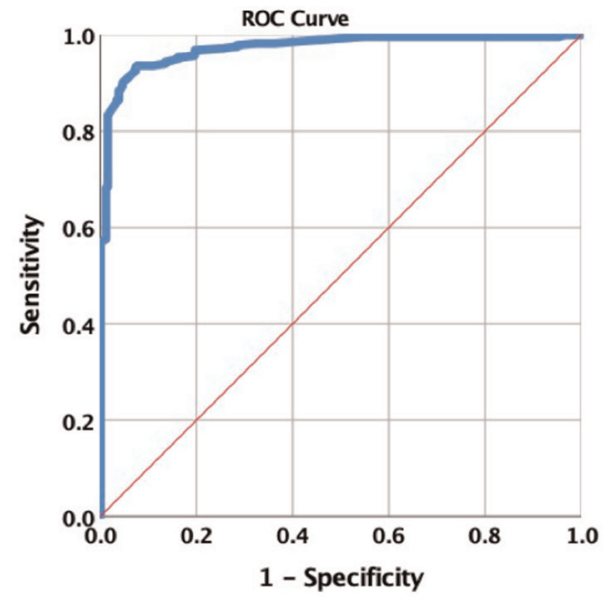

IEL Density for Coeliac
Introduction Intestinal epithelial cell damages generated by inflammation in coeliac disease (CD) ranges from sub-microscopic to severe architectural distortion. Translation of quantitative morphological changes in intestinal microorgans, like villus/crypt transformation, distribution of inflammatory cells and diagnostic cut offs, is lacking for $\mathrm{CD}$ and gluten related micro-enteropathies.

Method Investigators from 22 centres, 9 countries of 4 continents, recruited CD patients with Marsh 0-II histology $(n=299)$, NCGS $(n=151)$, and 262 controls. Based on an agreed protocol, epithelial morphology including intraepithelial lymphocyte (IEL) density, villus height and crypt depth were measured in well-oriented duodenal biopsies.

Results In total 712 subjects were recruited from Australia (20), Finland (20), India (25), Iran (37), Italy (246), Romania (10), Turkey (30), UK (166) and USA (158). Preliminary analyses showed raw IEL density (IEL/100EC) was poorly correlated with tTG, villus height, crypt depth or their ratios, and even significant findings did not show strong correlation coefficients $(<0.36)$. The IEL density cut off scored 93\% sensitivity and specificity at 24/100EC for CD. However, for NCGS the optimal sensitivity and specificity cut off was at 22IEL/ 100EC giving a sensitivity of $57 \%$ and specificity of $80 \%$ (see fig 1). The villus height was significantly shorter in CD compared to either control $(\mathrm{p}<0.001)$ or NCGS groups $(\mathrm{p}<0.001)$. Also, NCGS had short villus height than control $(\mathrm{p}<0.001)$.

Conclusion The most specific and strongest biomarker for CD with microenteropathy is serology acting as the gold standard in this group. Villus height and crypt depth would serve as complementary tools in diagnosis of mild CD and NCGS patients. NCGS seem to have a milder morphological change compared to $\mathrm{CD}$ even when they present with similar Marsh scores. This study also confirms the cut off of IEL for CD with microenteropathy is similar to $\mathrm{CD}$ with severe enteropathy at 25 IEL/100EC.

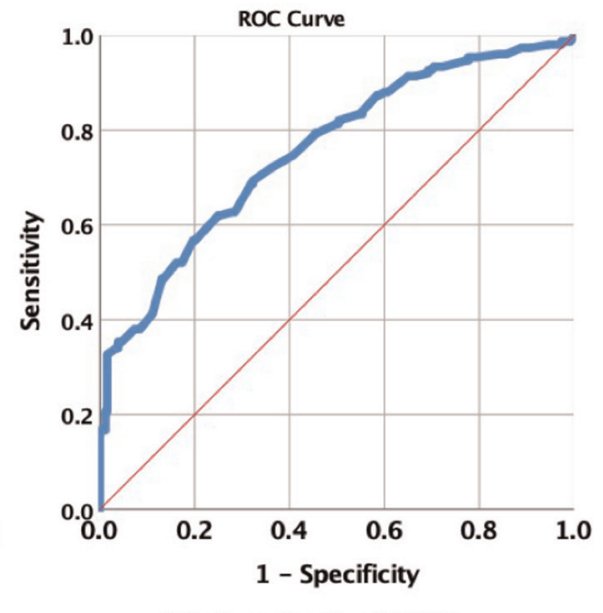

IEL Density for NCGS 